\title{
Synthesis of Hollow Conductive Polypyrrole Balls by the Functionalized Polystyrene as Template
}

\author{
Choo Hwan Chang, Pyung Soo Son, Jeong-Ah Yoon, and Seong-Ho Choi
}

Department of Chemistry, Hannam University, Daejeon 305-811, Republic of Korea

Correspondence should be addressed to Seong-Ho Choi, shchoi@hnu.kr

Received 26 September 2009; Accepted 16 March 2010

Academic Editor: Xiaogong Wang

Copyright (๑) 2010 Choo Hwan Chang et al. This is an open access article distributed under the Creative Commons Attribution License, which permits unrestricted use, distribution, and reproduction in any medium, provided the original work is properly cited.

We report the preparation of hollow spherical polypyrrole balls (HSPBs) by two different approaches. In the first approach, coreshell conductive balls, CSCBs, were prepared with poly(styrene) as core and polypyrrole (PPy) as shell by in situ polymerization of pyrrole in the presence of polystyrene (PS) latex particles. In the other approach, CSCBs were obtained by in situ copolymerization of pyrrole in the presence of PS(F) with hydrophilic groups like anhydride, boronic acid, carboxylic acid, or sulfonic acid, and then HSPBs were obtained by the removal of PS or PS(F) core from CSCBs. TEM images reveal the spherical morphology for HSPBs prepared from PS(F). The conductivity of CSCBs and HSPBs was in the range of $0.20-0.90 \mathrm{~S} / \mathrm{cm}^{2}$.

\section{Introduction}

Recently, conducting polymers have been actively studied due to their potential applications such as light emitting diodes, secondary batteries, electromagnetic interference (EMI) shielding, and antistatic coating $[1,2]$. Polypyrrole (PPy) is one of the most air-stable organic conducting polymers with a high conductivity. In addition, PPy is simple to prepare by oxidative polymerization.

However, PPy suffers from limited processability due to its insolubility in common solvents $[3,4]$. In order to improve the processability of $\mathrm{PPy}$, several research groups prepared colloidal dispersions of either pure PPy or PPycoated particles [5-7]. Polystyrene (PS) latex particles, stabilized by poly( $N$-vinylpyrrolidone) as the surfactant, were coated onto the surface of PPy by the in situ polymerization of pyrrole [8]. The thickness of PPy over the surface of PS latex particles has been controlled by varying the amount of PS in the latex. Cho et al. [9] reported the coating of nanosized PS latex particles with $\mathrm{PPy}$ via in situ polymerization. Surfactants were used as the anchoring agents to modify the hydrophobic properties of PS. To the best of our knowledge, reports are scarce on the preparation of PPy-coated PS latex particles without using a surfactant.
In this study, we report the preparation of hollow spherical PPy balls (HSPBs) by two different approaches. In the first approach, core-shell conductive balls (CSCBs), with polystyrene (PS) as core and PPy as shell, were prepared by in situ polymerization of pyrrole. The PS latex particles were covered with a layer of surfactant (sodium dodecyl sulphate (SDS) or poly vinyl pyrrolidone (PVP)) before the preparation of CSCBs. The surfactant modifies the hydrophobic surface of PS and helps in the formation of a layer of PPy over the surface of PS particles. The core (PS) was then removed from CSCBs to obtain the HSPBs. In the other approach, CSCBs were prepared by in situ polymerization of pyrrole in the presence of PS(F) spherical particles with anhydride or boronic acid carboxylic acid or sulfonate groups. Here again, the core (PS) and PS(F) were removed from CSCBs to obtain HSPBs. The morphology and size of the HSPBs were investigated by high-resolution transmission electron microscopy (HR-TEM) and fieldemission scanning electron microscopy (FE-SEM).

\section{Experimental}

2.1. Chemicals. Styrene (99\%), pyrrole (98\%), and potassium persulfate $\left(\mathrm{K}_{2} \mathrm{~S}_{2} \mathrm{O}_{8}\right)$ were obtained from Sigma-Aldrich 


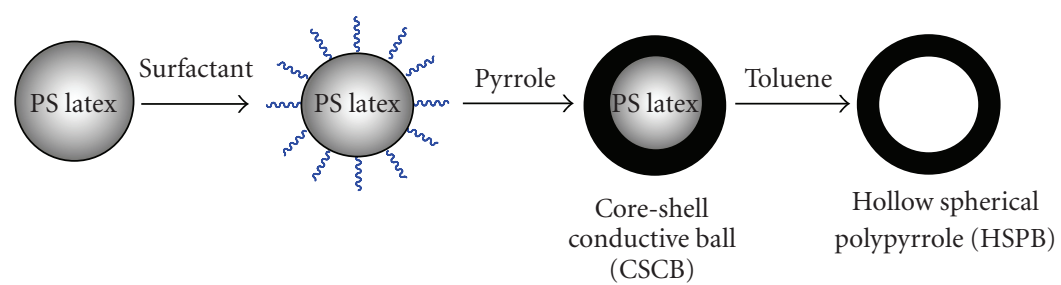

FIGURE 1: Preparation process of hollow conductive polymer ball by using surfactant as anchoring agent.
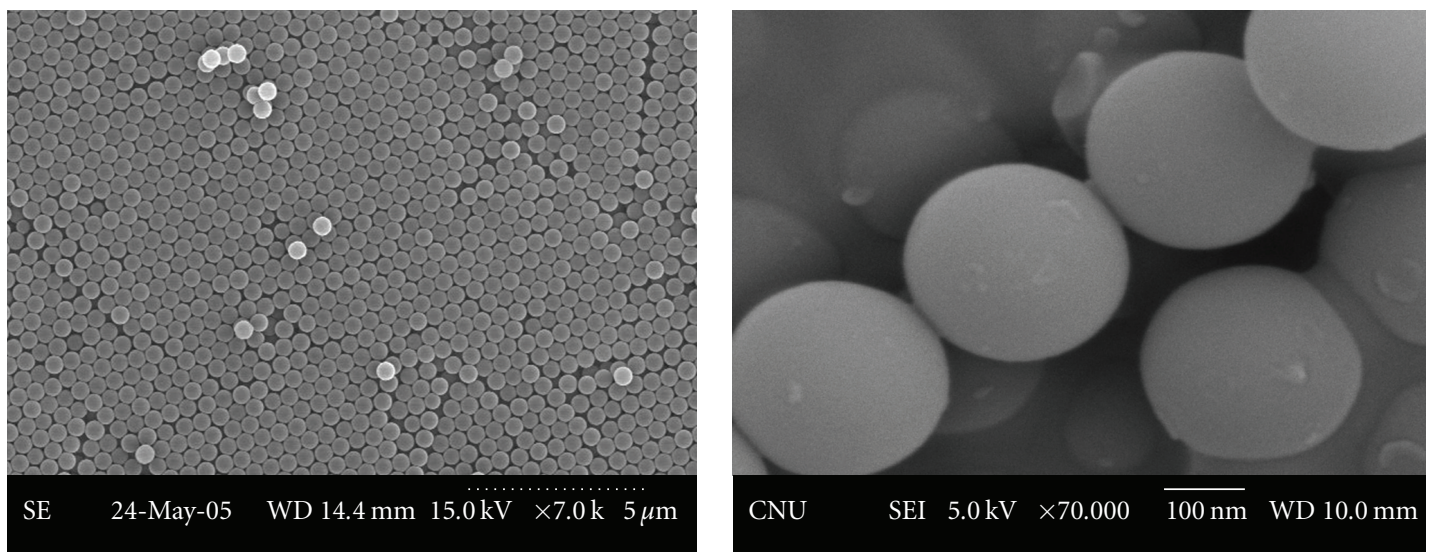

FIGURE 2: FE-SEM images of PS latex ball prepared by emulsifier-free emulsion polymerization.

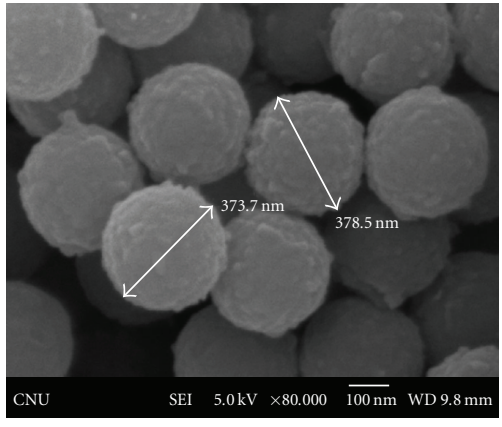

(a)

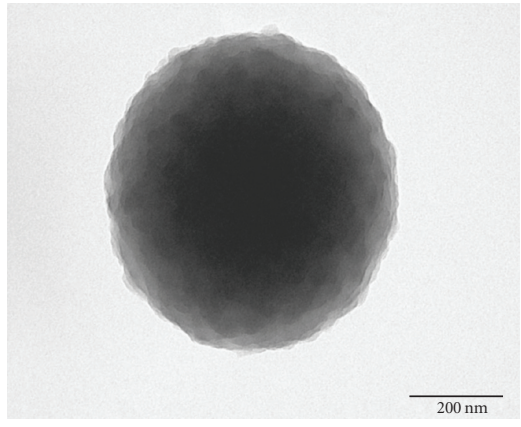

(b)

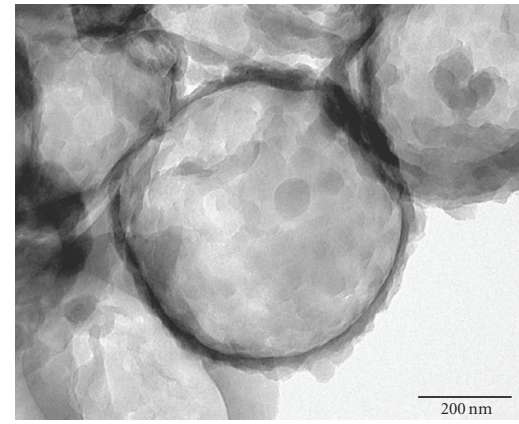

(c)

FIGURE 3: SEM and TEM images of CSCBs with core-PS and shell-PPy (a), (b), and TEM images of HSPBs (c). The SDS as anchoring agent was used for preparation of CSCBs.

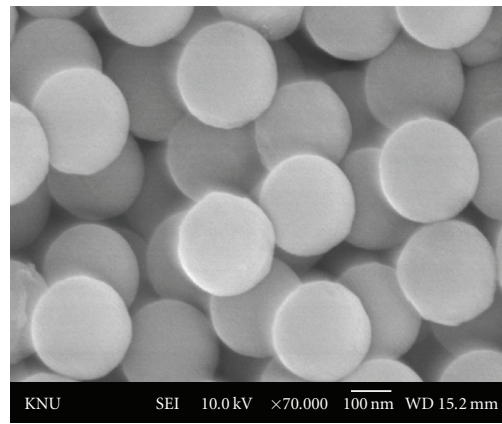

(a)

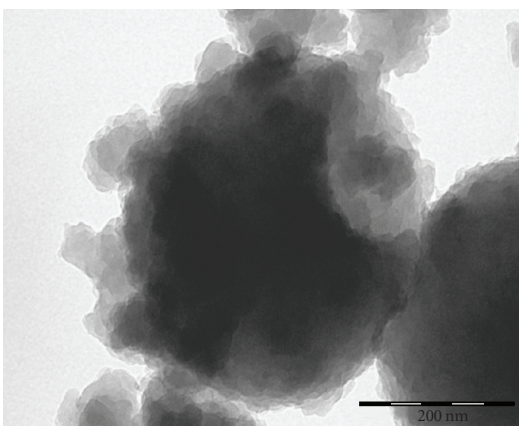

(b)

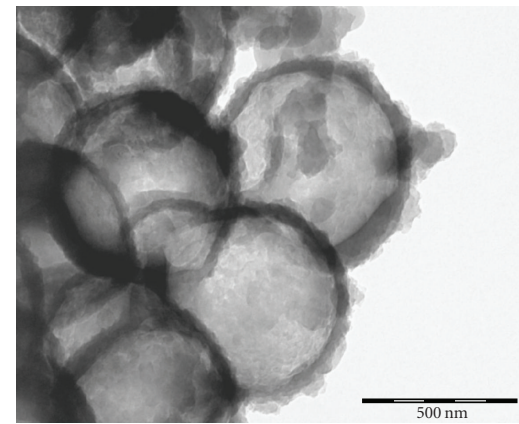

(c)

FIGURE 4: SEM and TEM images of CSCBs with core-PS and shell-PPy (a), (b), and TEM images of HSPBs (c). The PVP as anchoring agent was used for preparation of CSCBs. 


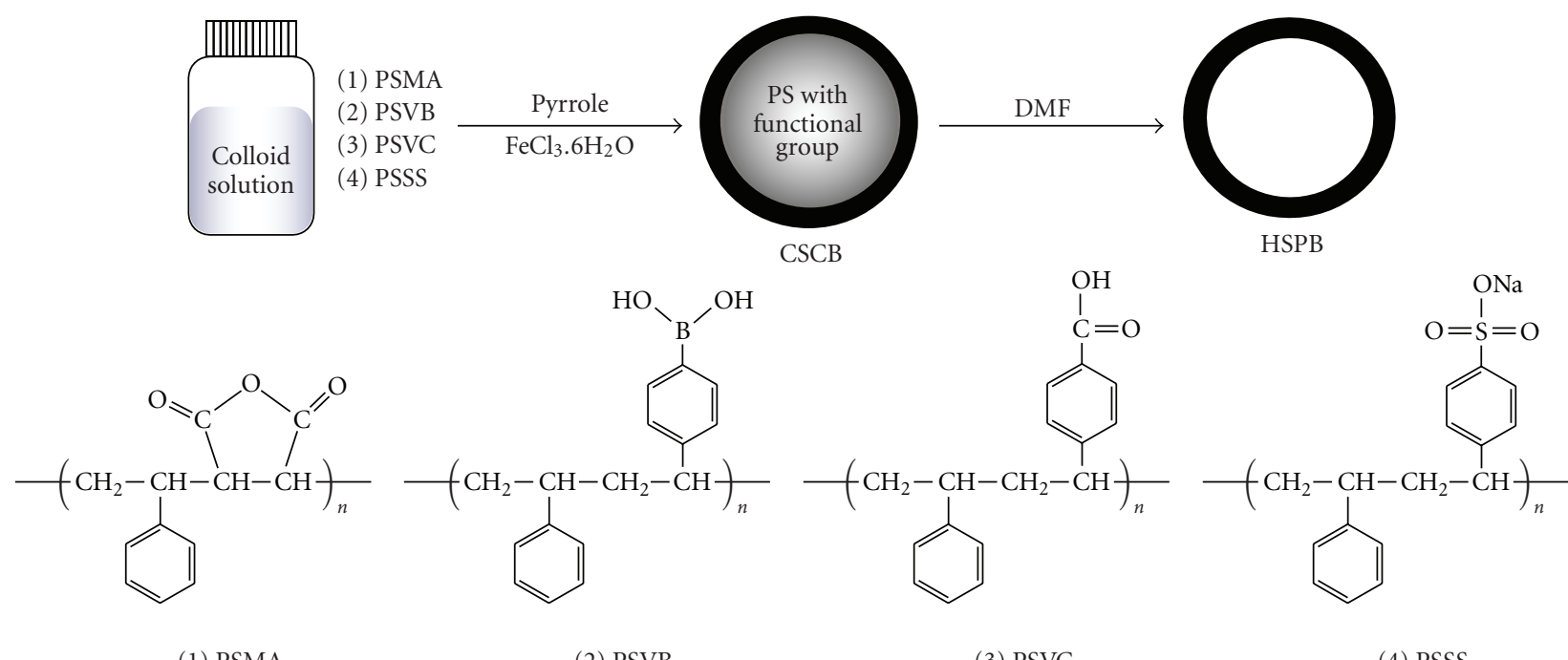

(1) PSMA

(2) PSVB

(3) PSVC

(4) PSSS

FIgURE 5: Preparation procedure of HSPBs without surfactant as anchoring agent.

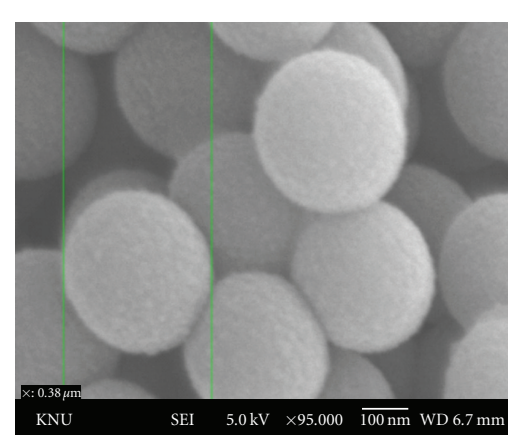

(a)

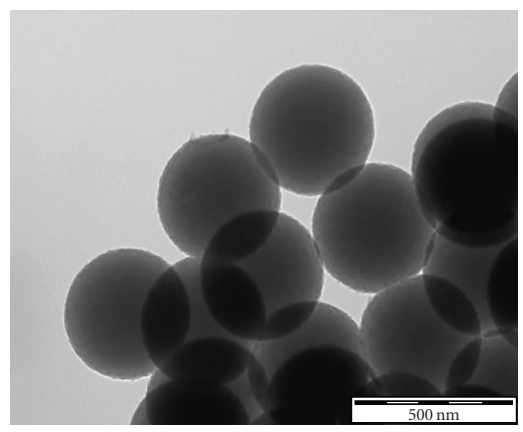

(b)

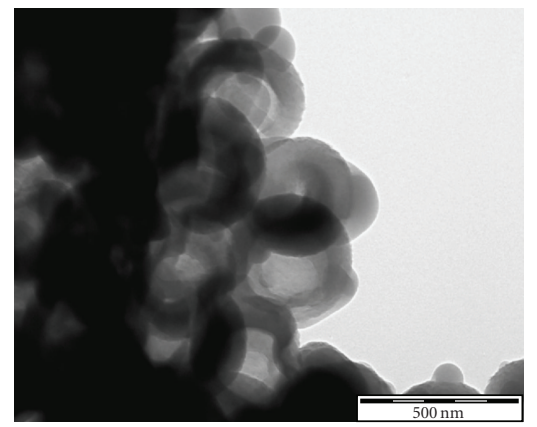

(c)

FIgURE 6: SEM and TEM images of CSCB with core-PSMA and shell-PPy (a), (b), and TEM image of HSPB (c).

Co. Sodium styrene sulfonate (NaSS), 4-vinylphenylboronic acid (VP), 4-vinylbenzoic acid (VB), poly (vinyl pyrrolidone) (PVP), sodium dodecyl sulphate (SDS), and methacrylic anhydride (MA) were purchased from Tokyo-Kasei (Japan). The $\mathrm{FeCl}_{3} \cdot 6 \mathrm{H}_{2} \mathrm{O}$ was obtained from Sam-Chunn Chemical Co.(Korea). All other chemicals were of reagent grades and used as such.

\subsection{Synthesis of PS Latex Particles and Functionalized PS} Particle $[P S(F)]$. The PS latex particles were prepared as follows. Styrene was mixed with an aqueous solution of potassium persulphate and polymerized at $75^{\circ} \mathrm{C}$ for $24 \mathrm{~h}$ by stirring at $350 \mathrm{rpm}$ under nitrogen atmosphere.

The functionalized PS particles, PS(F), were prepared as follows. Typically, the surface of poly(styrene) is as follows. An aqueous solution consisting $(700 \mathrm{~mL})$ of potassium hydroxide and potassium persulphate was prepared. Styrene $(45.3 \mathrm{~g}$ ) and 4-vinyl phenyl boronic acid $(1.00 \mathrm{gm})$ were then added to the above solution. In a similar way, PS(F) was also prepared with anhydride, carboxylic acid, and sulfonic acid groups with the respective reactant.

2.3. Synthesis of Hollow Spherical Polypyrrole Ball (HSPBs). HSPBs were prepared by two different approaches. In the first approach, the PS latex particles were used with coverage of a layer of the surfactant. PVP or SDS was used to modify the surface of PS. Typically, $0.4 \mathrm{~g}$ of PS latex particles was dispersed in an aqueous solution containing pyrrole and $10 \mathrm{~mL}$ PVP ( $0.1 \mathrm{~g})$. Polymerization was initiated by the addition of $\mathrm{FeCl}_{3}$ and continued for $24 \mathrm{~h}$. After polymerization, the precipitate, core- (PS-) shell (PPy) conductive balls (CSCBs), was centrifuged and dried in vacuum oven at $60^{\circ} \mathrm{C}$ for $8 \mathrm{~h}$. HSPBS were obtained by dispersing the CSCBs in toluene and stirring for $24 \mathrm{~h}$ (Figure 1).

In the other approach, PS(F) was used for the preparation of CSCBs. PS was functionalized with MA, VB, VP and to get PS (MA), and PS (VB), PS (VP), respectively. CSCBs were synthesized by the in situ polymerization of pyrrole in the presence of $\mathrm{PS}(\mathrm{F})$ using $\mathrm{FeCl}_{3}$ as the initiator. The HSPBs 


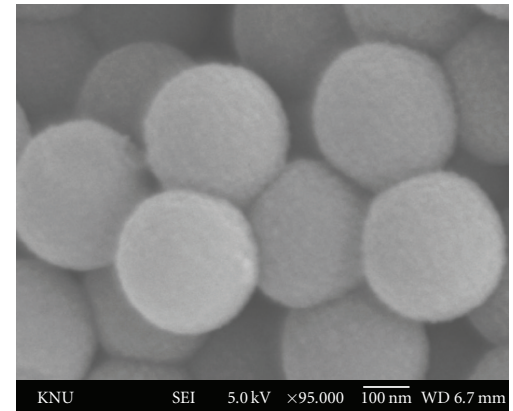

(a)

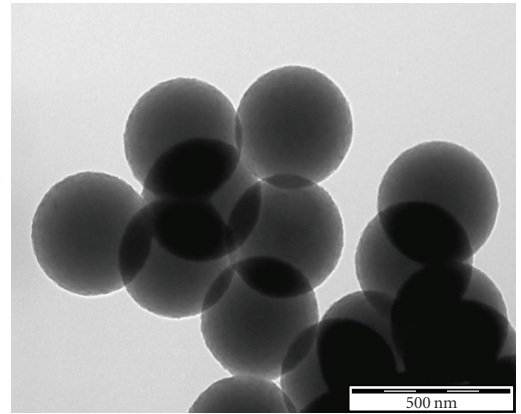

(b)

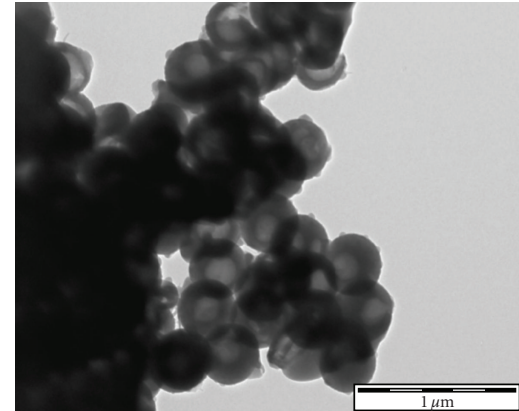

(c)

FIgure 7: SEM and TEM images of CSCBs with core-PSVB and shell-PPy (a), (b), and TEM image of HSPB (c).

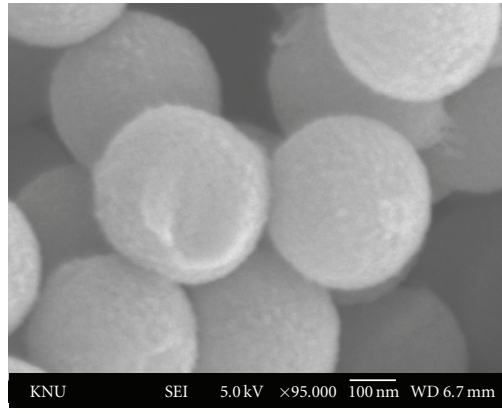

(a)

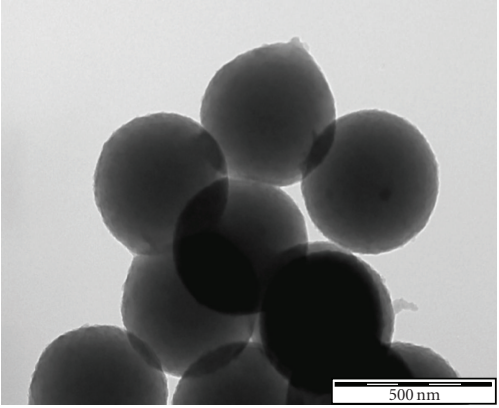

(b)

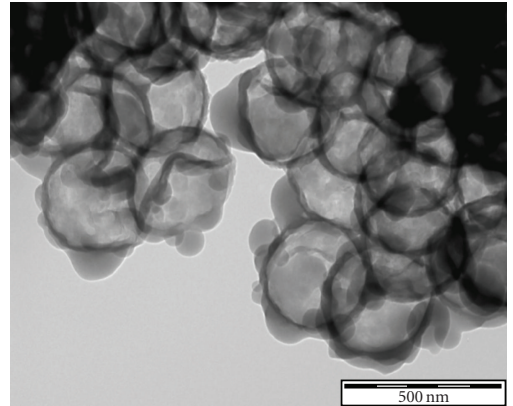

(c)

Figure 8: SEM and TEM images of CSCBs with core-PSVC and shell-PPy (a), (b), and TEM images of HSPBs (c).

were obtained by adopting a similar procedure as detailed above (Figure 2). The HSPBs were dried in vacuum oven at $60^{\circ} \mathrm{C}$ for $8 \mathrm{~h}$.

2.4. Analysis of CSCBs and HSPBs. Particle size and morphology of PS, PS(F) latex particles, CSCBs, and HSPBs were investigated by FE-SEM (Hitachi, S-4700, Japan) and HRTEM (JEOL, JEM-2010, USA). For the conductivity measurement, samples were prepared as follows. A conductive ink was prepared by mixing CSCBs or HSPBs $(15.0 \mathrm{mg})$ and poly(4-styrenesulfonic acid) $(150 \mathrm{mg})$ in a mortar and dissolving the mass in ethanol $(0.6 \mathrm{~mL})$. Subsequently, a slide glass was wet coated with the conductive ink by using a brush, and dried in vacuum oven at $60^{\circ} \mathrm{C}$ for $8 \mathrm{~h}$ under nitrogen gas. The conductivity was measured by 4 -point probe technique at room temperature (CMTSR3000/AIT). The conductivity was calculated using the following equations:

$$
R \times t=\rho, \quad \text { Conductivity }=\frac{1}{\rho}
$$

where the symbols $t$ and $\rho$ represent the thickness $(\mathrm{cm})$ and specific resistance $(\Omega \mathrm{cm})$, respectively.

\section{Results and Discussion}

FE-SEM images of PS latex particles are presented in two magnifications (Figure 3). The particles of PS are spherical with an average diameter of $\sim 345 \mathrm{~nm}$. Importantly, the surface of PS particles is generally hydrophobic. However, in the present case, PS latex particles are covered with a layer of the surfactant, SDS or PVP. Hence, it is presumed that the surface of PS latex particle is slightly hydrophilic due to the existence of anchoring agent (surfactant) on its surface. It is to be noted that the hydrophobic part of the surfactant is preferentially attached to the surface of latex PS particle. The hydrophilic end of the anchoring agent is present as the outer sheath. Thus, the PS latex particles are partially hydrophilic. Upon polymerization of pyrrole, the core (PS)-shell (PPy) spherical balls (CSCBs) were resulted.

SEM and TEM images of CSCBs (Figures 4(a) and 4(b)) and TEM images (Figure 4(c)) of HSPBs prepared from CSCBs which are prepared with SDS as the surfactant, are presented in Figure 3. The average diameter of CSCBs particles, prepared with SDS as the anchoring agent, is $380 \mathrm{~nm}$. This means that the shell thickness of PPy in CSCBs is $\sim 30 \mathrm{~nm}$. Figure 4 (c) presents the TEM image of few of the stable structure of HSPBs. HSPBs are found to have an average thickness of $\sim 30 \mathrm{~nm}$.

Figure 5 presents the SEM (Figure 5(a)) and TEM (Figure 5(b)) images of CSCBs prepared using PVP as the stabilizing agent. In this case, a nonuniform layer of PPy 


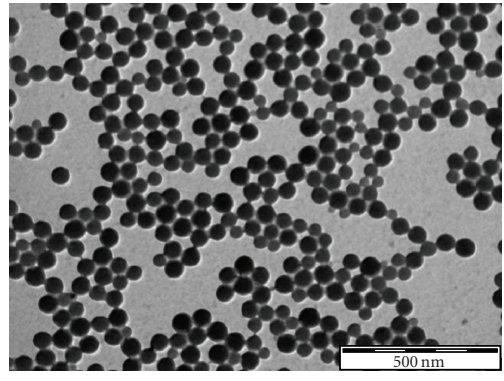

(a)

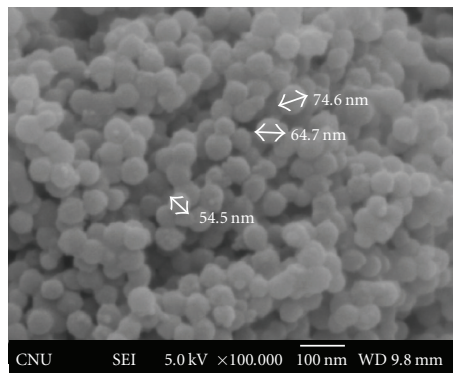

(b)

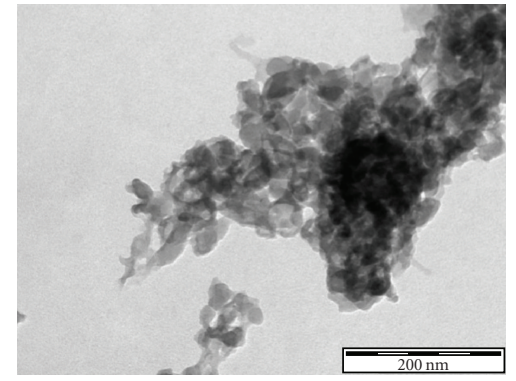

(c)

FIGURE 9: TEM images of PSSS (a), SEM images of CSCB with core-PSSS and shell-PPy (b), and TEM images of HSPB (c).

TABLE 1: Conductivity of the CSCBs and HSPBs by a standard 4-point probe technique at room temperature ${ }^{(a)}$.

\begin{tabular}{|c|c|c|c|c|c|}
\hline CSCBs with core-PS ${ }^{(\mathrm{b})}$ & CSCBs with core PSMA & CSCBs with core PSVB & CSCBs with core-PSVC & CSCBs with core-PSSS & $\mathrm{HSPBs}^{(\mathrm{c})}$ \\
\hline $0.3 \mathrm{~s} / \mathrm{cm}^{2}$ & $0.20 \mathrm{~s} / \mathrm{cm}^{2}$ & $0.40 \mathrm{~s} / \mathrm{cm}^{2}$ & $0.67 \mathrm{~s} / \mathrm{cm}^{2}$ & $0.90 \mathrm{~s} / \mathrm{cm}^{2}$ & $0.25 \mathrm{~s} / \mathrm{cm}^{2}$ \\
\hline
\end{tabular}

(a) Poly(4-styrenesulfonic acid ) was used as a dopant.

(b) The CSCBs with core-PS and shell PPy were prepared using SDS as anchoring agent.

${ }^{\text {(c) }}$ The HSPBs were measured in Figure 3.

could be seen with an average layer thickness of $\sim 50 \mathrm{~nm}$. Figure 5(c) presents the TEM images of HSPBs obtained from CSCBs (PVP). TEM image also reveals that the PPy layer in HSPBs is not uniform. Thus, it is evident that coating or anchoring with surfactant over PS could not result HSPBs with a uniform layer of PPy.

An alternative approach of using functionalized PS particles to prepare PS(F) was adopted. The functional groups that are present on the surface of PS (F) are expected to have stronger interactions with PPy. Functional groups like carboxylic acid, boronic acid, anhydride, and sulfonic acid groups are selected to form PS(F) (see, Figure 2). Large amount of PPy molecules are expected to be bound over the surface of PS(F). Also, a compact layer of PPy is expected in CSCBs prepared with $\mathrm{PS}(\mathrm{F})$ as core.

Figures 6, 7, 8, and 9 show SEM and TEM images of CSCBs prepared with PSMA, PSVB, PSVC, or PSSS as core with PPy as the shell. CSCBs prepared with PS(F) have a stable spherical morphology as compared to the CSCBs prepared with PS (surfactant). The shell thickness of PPy in the HSPBs prepared from CSCBs of PS(F) is much higher $(>50 \mathrm{~nm})$ as compared to shell thickness of PPy in CSCBs prepared from PS (surfactant) except for PS(F) with sulfonic acid.

Table 1 summarizes the conductivity of the CSCBs and HSPBs. The conductivity of CSCBs and HSPBs was in the range of $0.20-0.90 \mathrm{~S} / \mathrm{cm}^{2}$ and indicates conducting nature of CSCBs and HSPBs. Thus, the CSCBs and HSPBs could be used as a coating material to cover the surface of an insulating polymer, and such a composite can find fluid applications in electromagnetic interference (EMI).

\section{Conclusion}

This study describes the preparation of hollow spherical polypyrrole ball (HSPBs) with high conductivities. Among the methods to obtain HSPB, the one which involves the use of functionalized PS particles seems to be promising for the preparation of stable and uniformly spherical HSPBs. This methodology may be extended for the preparation of hollow spherical balls of other conducting polymers.

\section{Acknowledgments}

This study is supported by the Nano R\&D Program via the Korea Science and Engineering Foundation funded by the Ministry of Science and Technology. In particular, the authors thank the Hannam University Research Fund (2010).

\section{References}

[1] V. Sittinger, A. Pflug, W. Werner, et al., "Production of MF and DC-pulse sputtere anti-reflective/anti-static optical interference coatings using a large area in-line coater," Thin Solid Films, vol. 502, pp. 175-180, 2006.

[2] N. Al-Dahoudi, H. Bisht, C. Göbbert, T. Krajewski, and M. A. Aegerter, "Transparent conducing, anti-static and anti-staticanti-glare coatings on plastic substrate," Thin Solid Films, vol. 392, pp. 299-304, 2001.

[3] Y. Lu, A. Pich, and H. Adler, "Synthesis and characterization of nanometer-sized polypyrrole composites," Synthetic Metals, vol. 135-136, pp. 37-38, 2003.

[4] W. Yin, J. Li, Y. Li, J. Wu, and T. Gu, "Conducting composite film based on polypyrrole and crosslinked cellulose," Journal of Applied Polymer Science, vol. 80, no. 9, pp. 1368-1373, 2001.

[5] F. Yang, Y. Chu, S. Ma, Y. Zhang, and J. Liu, "Preparation of uniform silica/polypyrrole core/shell microspheres and polypyrrole hollow microspheres by the template of modified silica particles using different modified agents," Journal of Colloid and Interface Science, vol. 301, no. 2, pp. 470-478, 2006. 
[6] D. B. Cairns, M. A. Khan, C. Perruchot, A. Riede, and S. P. Armes, "Synthesis and characterization of polypyrrolecoated poly(Alkyl Methacrylate) latex particles," Chemistry of Materials, vol. 15, pp. 233-239, 2003.

[7] L. Hao, C. Zhu, C. Chen, et al., "Fabrication of silica coreconductive polymer polypyrrole shell composite particles and polypyrrole capsule on monodispersed silica templates," Synthetic Metals, vol. 139, no. 2, pp. 391-396, 2003.

[8] S. Bousalem, A. Yassar, T. Basinska, et al., "Synthesis, characterization and biomedical applications of functionalized polypyrrole-coated polystyrene latex particles," Polymers for Advanced Technology, vol. 14, pp. 820-825, 2003.

[9] S.-H. Cho, W.-Y. Kim, G.-K. Jeong, and Y.-S. Lee, "Synthesis of nano-sized polypyrrole-coated polystyrene latexes," Colloids and Surfaces A, vol. 255, no. 1-3, pp. 79-83, 2005. 

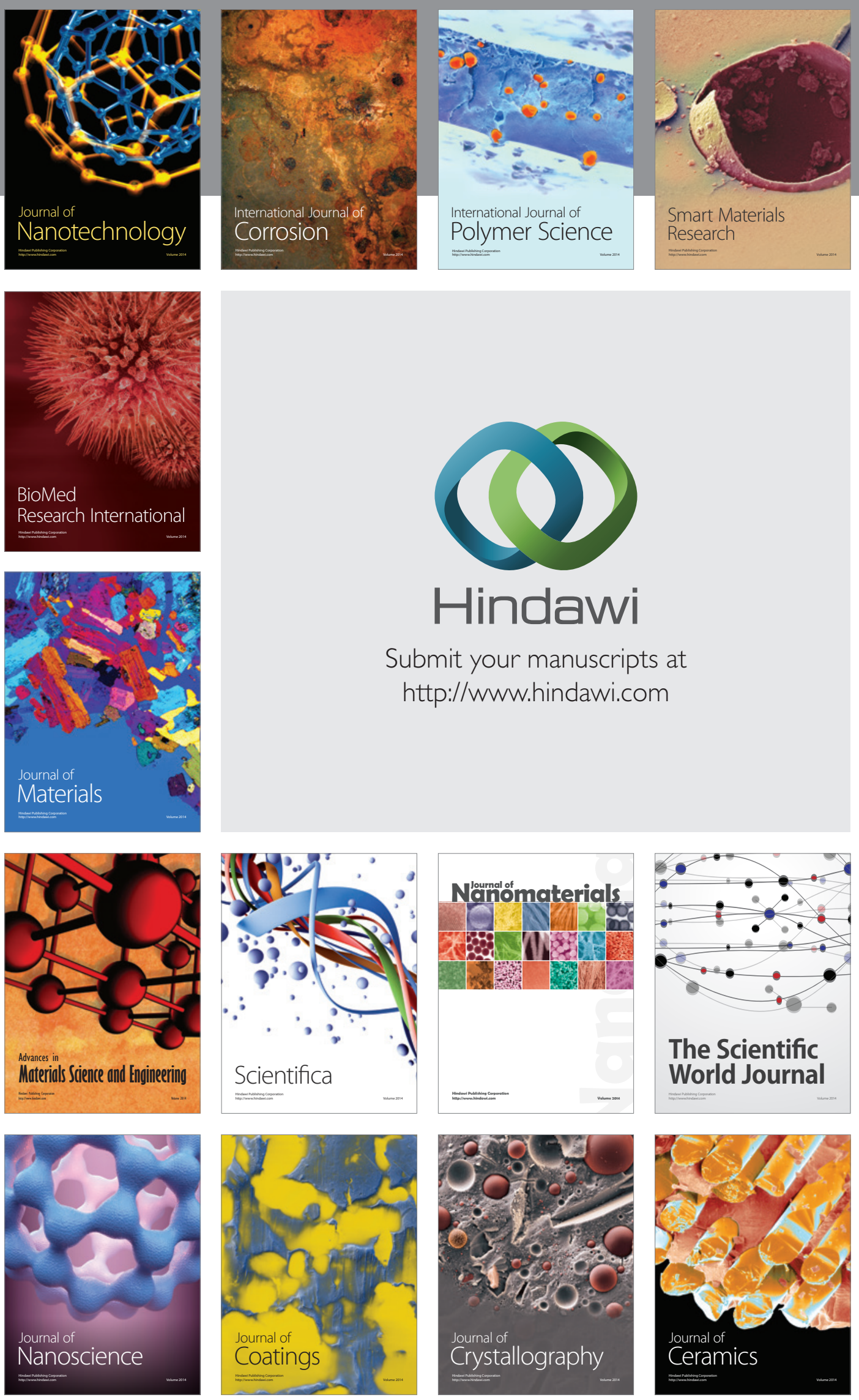

The Scientific World Journal

Submit your manuscripts at

http://www.hindawi.com

\section{World Journal}

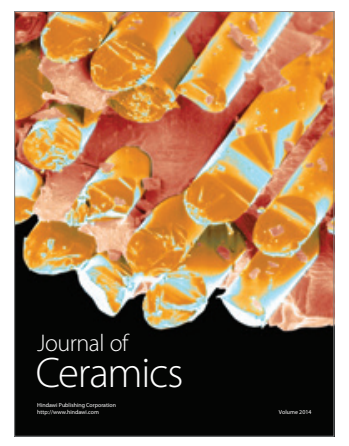

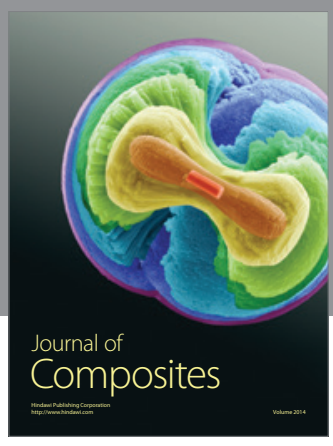
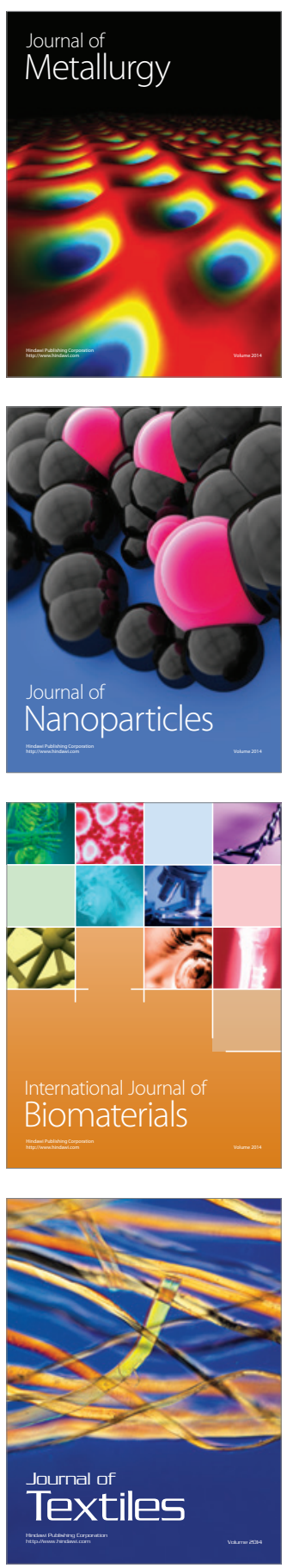